\title{
PATRÓN DE CRECIMIENTO DE LA ECONOMÍA PERUANA
}

\section{PATRÓN DE CRECIMIENTO}

\subsection{Patrón de Crecimiento Económico del Periodo: 1985 - 1990}

La política de estabilización económica que inició el gobierno de Alan García estuvo marcada por dos influencias principales. La primera fue la idea de la "pirámide social", cuyo análisis se basaba en la idea de una gran pirámide cuyos niveles representaban los distintos estratos de la sociedad peruana, y la segunda, fue la experiencia del Plan Austral, que en aquel momento estaba dándole grandes réditos políticos al presidente de Argentina Raúl Alfonsín.

"(...) Lo que el Perú necesita es una revolución democrática, una reestructuración histórica que reactive lo profundo, que desmordace las fuerzas sociales hasta hoy ignoradas. Propongo impulsar la agricultura donde millones de peruanos viven como hace siglos, para que esas tierras produzcan los alimentos que ahora compramos en el extranjero. Esa será la reactivación social productiva. Propongo, en segundo lugar, que los cientos de miles de brazos desempleados y subempleados de los pueblos jóvenes, tengan algún acceso al ingreso o empleo. Esa será la reactivación social del consumo (...)".(García Pérez, 1985).

Para resumir la estructura productiva del país, se puede decir que la política de reactivación se iba a basar en la articulación entre el sector informal urbano y el sector rural andino. Así pues, de acuerdo a este enfoque de la reactivación, se podía suponer que la reactivación industrial debía esperar a que el dinamismo "desde abajo" operara sobre el conjunto de la economía.

El segundo factor que configuró la política de estabilización fue la influencia de la heterodoxia. Para algunos, la heterodoxia fundamentaba un programa estabilizador "(...) que intenta una política económica consistente para disminuir la inflación a cero con neutralidad distributiva. Este último elemento es la característica central que le da a la heterodoxia su especificidad" (Espejo, 1989).

La interpretación del proceso inflacionario se enmarcó en la concepción neoestructuralista: "Las alzas en el nivel general de los precios se explican a partir de decisiones que influyen directamente en el costo de los bienes y servicios. En un enfoque de tal clase, la inflación se explica por cambios en el comportamiento de los actores (asalariados, empresarios, rentistas y gobierno) que sean capaces de influir básicamente sobre los cuatro precios básicos: el nivel del salario, el margen de utilidad sobre costos primos o markup, la tasa de interés y el tipo de cambio". (Carbonetto y otros, 1987).

Según el neoestructuralismo los precios del sector oligopólico industrial se forman de acuerdo a los costos de insumos importados, los costos laborales y los precios controlados. De allí se concluye que el nivel de precios en el sector moderno depende del tipo de cambio, de los salarios y de los precios controlados nominales. En otras palabras, la tasa de inflación depende de la tasa de devaluación; la tasa de crecimiento 
de los precios controlados y la tasa de crecimiento de los salarios nominales.

De acuerdo a este contexto se aplicaron ciertas medidas económicas con el fin de superar los problemas que afectaban al país y lograr los objetivos trazados. Así el Plan de Emergencia aplicado en julio de 1985 tuvo un carácter fundamentalmente heterodoxo, pero también incluyó algunos ingredientes ortodoxos, lo cual reflejaba la existencia de una cierta cautela relativa al manejo fiscal y al sector externo.

\subsection{El Plan de Emergencia aplicado en julio de 1983}

Entre las medidas de corte heterodoxo aplicados por el gobierno de García Pérez para superar la crisis heredada se encuentran:

- La congelación de todos los precios. La congelación debía cumplir un papel coordinador de precios, propios de ensayos heterodoxos. Se esperaba que no hubiera inflación de ahí en adelante, siendo cualquier aumento en algún precio señal de un incremento en el margen de ganancia. Por ello, el gobierno decreto la congelación de todos los precios de los bienes y servicios y alquileres a los niveles en que se encontraban el día 27 de julio de 1985.

- Devaluación de la moneda y reducción de la tasa de interés. En el marco de la teoría no ortodoxa la reducción de la tasa de interés no debía afectar al ahorro, en tanto éste no depende de ella, sino fundamentalmente, del nivel del ingreso generado por la mayor rentabilidad de las actividades productivas.

- Incremento en las remuneraciones. La posibilidad de reducir la inflación sin reducir la capacidad adquisitiva de los trabajadores, era según versiones del gobierno, uno de los grandes deslindes respecto a los ajustes ortodoxos del pasado.

Estas medidas conformaron el eje principal del programa económico en la cual "el aumento de los costos de insumos importados ocasionado por la devaluación y el aumento otorgado a los trabajadores será compensado por la reducción de la tasa de interés efectiva (...). Pero esta medida se orienta también a que fundamentalmente, se logre una redistribución en la estructura de costos de las empresas que implique trasladar recursos de las actividades netamente especulativas, alentadas por las altas tasa de interés, hacia los sectores productivos, lo que a su vez, permitirá un crecimiento del consumo interno" (Alva Castro, 1985).

Este programa económico tuvo, adicionalmente, ciertos rasgos que parecen ser exclusivos del experimento heterodoxo peruano. El primero radica en la política de congelación de precios, que a diferencia de otras experiencias de estabilización no ortodoxas, afectó a un conjunto bastante amplio de bienes. Un segundo rasgo fue el tratamiento de la deuda externa, del cual sólo se destinó al servicio de la deuda el 10 por ciento del valor total de las exportaciones.

Los otros rasgos complementarios fueron:

- La elevación de la tasa de cambio real. Debido a que una reactivación de la economía requería un ajuste expansivo de la Balanza de Pagos, para lo cual era conveniente mantener una tasa de cambio efectiva por lo menos de paridad o ligeramente superior. (Alva Castro, 1985).

- Se incrementaron los impuestos en los combustibles en vez de una emisión inorgánica para financiar el déficit fiscal. (La 
estructura tributaria descansaba fuertemente en el impuesto al combustible).

- La restricción monetaria. El Banco Central, buscando contrarrestar el efecto monetizador de la dolarización, estimuló una fuerte contracción de la liquidez del sistema bancario, no sólo elevando la tasa de encaje marginal sino pagando una alta remuneración al sobreencaje. Se redujo así el estímulo para otorgar créditos al sector privado.

En síntesis, se puede decir que los principales resultados inmediatos del Programa de Emergencia de agosto de 1985 fueron la reducción de la inflación, el impacto recesivo inicial, la desdolarización, el mantenimiento del superávit comercial externo y la elevación de RIN, la probable neutralidad distributiva, la reducción del déficit fiscal respecto del PBI, y una mejora de los términos de intercambio a favor de la agricultura.

La política aprista en el periodo posterior al ajuste (octubre 1985) no sólo pretendió elevar la demanda, a través del consumo, para aumentar la producción sino que simultáneamente buscó mejorar la distribución del ingreso a favor de los asalariados. Existiendo capacidad instalada ociosa; y empleando más la capacidad instalada, más trabajadores, los costos unitarios se reducirán.

Para esos efectos se dictaron un conjunto de medidas tendientes a reactivar la economía. Entre las que destacan eliminación del IGV sobre diversos productos, la reducción de la tasa de interés, el otorgamiento de facilidades a empresas para aumentar los turnos de trabajo, la concesión de reintegros tributarios a los exportadores; la exoneración de pagos de derechos arancelarios y de impuestos a la importación de maquinaria; la creación de líneas de crédito preferencial, etc.
Por otro lado, el otorgamiento de préstamos a los trabajadores del sector público y la puesta en marcha de un programa de empleo masivo debían impulsar la demanda interna.

"El estado mantendrá como objetivo el control, teniendo a la regulación y concertación de los precios en aquellos casos en los que, a pesar de las medidas, el costo de producción resulte mayor que en los precios de venta. Lo importante de una política de regulación de precios es que éstos no aumenten más que el incremento de los salarios. El estado repartirá los recursos en las actividades productivas. La primera forma de impulsar la producción es aumentando la demanda, sin que esto signifique trasladar a los costos de producción el aumento de los salarios (...). Debemos estimular en estos meses la capacidad de consumo y de compra de nuestros trabajadores".(García Pérez, 1985).

El consumo de la clase asalariada se convirtió en la clave de la posibilidad de reactivar la economía: era dicho consumo el encargado de ampliar el mercado y encabezar el crecimiento inmediato.

Se instaló así la reactivación del consumo. Asimismo; si por un lado, los asalariados se encargaran de ampliar el mercado; por el otro, eran los empresarios privados quienes debían encargarse de elevar la inversión.

La lentitud de la reactivación y el desabastecimiento de alimentos a principios de 1986, obligó al gobierno a aplicar en febrero de 1986 un nuevo paquete de medidas para impulsar la producción. El consumo privado surge como el principal elemento explicativo de la recuperación de la demanda y el producto agregado. 
El Plan de Emergencia cuyos objetivos principales fueron combatir la inflación establece: "Creemos que para el crecimiento real de la agricultura, que es la mejor garantía de la descentralización y la democracia, es necesario hacer que la producción agraria sea rentable. Por eso el Estado ha destinado la suma de tres billones de soles como Fondo de Garantía para asegurar al campesinado un precio mínimo que le permita supervisar y ampliar su capacidad productiva. Igualmente (...) va a ampliarse largamente ese volumen de crédito, reduciéndose una vez más el interés que cobra el banco, para impulsar la producción de alimentos (...). Pero es en el sector industrial, en el sector urbano, donde están las mayores posibilidades de crear nuevo empleo (...) confiamos lograr un impulso de crecimiento industrial ampliando y ayudando también a la exportación no tradicional. En conclusión, por la ampliación de la demanda, la regulación de los precios, la reducción de los costos de producción, el mantenimiento de la tasa de cambio (...) y la limitación del servicio de la deuda, estamos planteando el impulso de una política de crecimiento para todos los sectores, para la agricultura, industria, los gastos sociales y productos del Estado". (García Pérez, 1986).

Las reservas del sistema bancario, que hasta marzo de 1986 habían crecido gracias a la desdolarización y a la adquisición acelerada de dólares, empezaron a declinar de manera contínua, agudizándose su caída desde mediados de 1987.

A mediados de 1986 se acabó el superávit comercial externo. A partir del último trimestre de 1986 la política económica apuntó a contrarrestar esa tendencia mediante medidas de contracción de la demanda. Esto restringió las importaciones y comenzó a generar un superávit comercial, pero durante el segundo semestre de 1987, la expansión de la demanda interna por manufacturas, la fuerte caída del tipo de cambio real para la importación y las expectativas de futuras devaluaciones elevaron bruscamente las importaciones llegándose a un déficit comercial que continuó hasta el tercer trimestre de 1988.

El temor a una presión de demanda durante el verano de 1987 hizo que se posterguen los aumentos de remuneraciones, lo que deterioro la capacidad adquisitiva de las remuneraciones sujetas a aumentos oficiales. El aumento en abril de ese año en el sector público no permitió recuperar la capacidad adquisitiva pérdida. Las remuneraciones reales desde entonces y hasta setiembre de 1988 tuvo un ritmo oscilante y ligeramente declinante.

En 1987, se observaron tasas de crecimiento de los precios controlados mayores a las de 1986, aunque esto aún no se aplicaba a los precios no controlados. A partir del segundo trimestre de 1987 se flexibilizó el control de precios, pasándose varios productos de la categoría de precios regulados a la de supervisados. Pese a los intentos de reajustar los precios públicos, el resultado final fue el mantenimiento de una brecha todavía grande entre el aumento de los precios controlados, el IPC (índice de precios al consumidor) y el de los no controlados.

El problema fiscal acompañó al externo durante 1987. Los recortes de los impuestos indirectos como parte de la estrategia antiinflacionaria y reactivadora, así como de de los impuestos a la exportación, y el que la recaudación por impuestos directos no aumentara con la reactivación, contrajeron los ingresos tributarios. Entre las explicaciones de ese creciente problema fiscal están el subsidio cambiario, la descapitalización de las empresas públicas y la política de precios. 
En 1987, las tasas de interés del sistema financiero fueron modificadas en dos oportunidades, configurándose a partir de julio una estructura de tasas activas con niveles inferiores a las de 1986 para operaciones de corto y mediano plazo.

En resumen, puede afirmarse que durante 1987 se registró una desaceleración del crecimiento de la producción, un deterioro del balance comercial, una caída de las reservas internacionales, una gran reducción en la capacidad instalada ociosa, un crecimiento de la tasa de inflación colectiva, una caída en la recaudación tributaria con el consecuente aumento del défrcit fiscal, y el fin de la remonetización iniciada al cambio de gobierno.

En setiembre de 1988, la economía entró en crisis. "No puede aumentar el consumo privado, la inversión privada y el producto (...) si las exportaciones descienden en términos reales. Existen asimismo brechas peligrosas en la economía: la brecha cambiaria, fiscal, desequilibrios en el mercado cambiario. Todo ello condujo al desarrollo de un proceso inflacionario que no puede ser atacado con medidas tibias y graduales, el gradualismo y la indexación de precios de la economía pueden conducir peligrosamente a un fenómeno de hiperinflación con recesión". (Salınas, 1988).

Se anunció así un programa de ajustes a corto plazo cuyos objetivos fueron tres: detener el proceso inflacionario y recesivo, terminar con la pérdida de divisas, y crear un contex to de estabilidad y confianza que permita un normal desempeño de los agentes económicos.

\subsection{Después de la Heterodoxia el Programa de Ajuste}

El programa de ajuste tuvo dos partes:
- La primera correspondía a las medidas destinadas a cerrar la brecha cambiaria y fiscal, con el fin de que el Banco Central pudiera reducir drásticamente su emisión inorgánica. Otro objetivo era ganar suficiente paridad cambiaria a fin de que el plan no afecte los niveles de exportación. $\mathrm{La}$ concepción de esta primera era esencialmente ortodoxa, en tanto se buscaba el realineamiento de precios relativos con reducción de la demanda agregada y el cierre de brechas.

- La segunda parte del programa era heterodoxa. Consistía en un congelamiento de precios (salarios, tipo de cambio, precios públicos, tarifas y tasas impositivas) por 120 días, a contar tras diez días de ajustes privados. Se congelarían así los costos de producción y consecuentemente los precios finales, por lo que el gobierno proyectaba que se reduciría la inflación. Sin embargo, el gran incremento de los precios del sector privado durante los diez días previos al congelamiento imposibilitó sostener el programa, viéndose obligado el gobierno a suspenderlo el 21 de setiembre. Así, la propia decisión de otorgar diez días para el realineamiento de precios, empujó a las empresas a grandes aumentos, pensando en los niveles que requerirían a fines de año. El temor del sector privado a perder ingresos ante la inflación, redundó en un comportamiento defensivo que contribuyó al resultado inflacionario en setiembre.

Por otro lado, la liberalización de precios tras suspender el congelamiento fue parcial, pues hubo precios controlados que siguieron congelados, correspondientes principalmente a las tarifas de servicios públicos. La coexistencia de precios liberados con otros congelados, en el contexto especulativo y de incertidumbre que enmarcó al "Plan de Setiembre", hacía previsible la necesidad de más correcciones, 
como las que se dieron en noviembre de ese año, las bases para una reactivación de la producción. Para preservar la solidez de las cuentas externas se tomaron medidas arancelarias y para-arancelarias, que a su vez tenían como objetivo proteger la industria nacional. En cuanto a las exportaciones se aumentó el financiamiento FENT (Fondo para las Exportaciones No Tradicionales), así como el porcentaje de libre disponibilidad, a negociarse en el Mercado Financiero. Posteriormente, hacia fines del año 1985, se establecieron modificaciones tributarias, orientadas a reordenar y simplificar el sistema tributario.

En forma complementaria y dentro de la estrategia de recuperación de la producción a través del incentivo de la demanda, fue necesario mejorar la capacidad del poder adquisitivo de la población, para lo cual se procedió a incrementar el ingreso mínimo vital y a reajustar las remuneraciones de los trabajadores. El mayor costo por estas medidas fue compensado por la reducción en los costos financieros, derivada de las disminuciones de la tasa de interés, y la presión de las expectativas fue reducida mediante la congelación de precios y la fijación del tipo de cambio.

\subsection{Patrón de Crecimiento Económico en el Periodo: 1991 - 1996}

Tras el fracaso del modelo heterodoxo impuesto por el gobierno pasado, el actual gobierno del Presidente Fujimori, inició un modelo económico que se ubica en el extremo monetarista de un conjunto de opciones de estrategias a las cuales los países en desarrollo podrían recurrir.

Este modelo económico experimentado en 1973, le está dando grandes resultados al país sureño. Pero tanto en Chile como en nuestro país, para que la aplicación de este modelo se diera, tuvo que estable- cerse un régimen autoritario (salvando las diferencias) a cuyo amparo se desarrolló el modelo monetarista vigente. Bajo ese régimen, los ejecutores del modelo económico han dispuesto de una autonomía excepcional para diseñar, poner en práctica y ajustar su acción.

Es indiscutible que en agosto de 1990 prevalecía en el Perú, substanciales desequilibrios macroeconómicos que era indispensable corregir. Asimismo, la economía estaba sobreintervenida, con excesivos controles microeconómicos sobre empresas privadas y públicas. La magnitud de los desequilibrios y la inorganicidad del intervencionismo público facilitó, aún sin justificación, que el enfoque monetarista ortodoxo se abriese camino.

Antes de desarrollar el modelo resulta conveniente definir los elementos básicos de este. En términos sintéticos, el modelo económico es un modelo capitalista con empresas privadas y con un sistema de laissez faire completamente integrado a la economía mundial, esto quiere decir, un sistema económico de economía privada con mercados libres, libre comercio, libre movilidad de capitales financieros, con reglas neutras y mecanismos automáticos que resuelven cualquier problema económico.

En respuesta a la aplicación del modelo se registraron cambios substanciales en el rol desempeñado por el sector público y privado en la actividad económica:

- El sector privado sería el principal y eventualmente el único productor. La empresa privada y la iniciativa personal constituirán el motor del crecimiento económico.

- El Estado y las empresas públicas • eran por definición ineficientes en lo económico. En consecuencia, mientras 
menor la participación del sector público en la actividad económica, esto implicaría un incremento de bienestar para la sociedad.

El ideal sería que el Estado se dedicara a resguardar la ley y el orden y que se abstuviera de intervenir en la cuestión económica. Es así como se impulsa el programa de privatización total de la economía y se habla de que hay que privatizar los caminos, la educación, la salud, etc.

-En su comportamiento económico, el sector público tiene que someterse a dos reglas básicas:

-Eliminación total del déficit y total autofinanciamiento de todas las empresas públicas.

-Reducción al mínimo del endeudamiento extemo. De esta manera, se eliminaría el problema de crisis de Balanza de Pagos.

- Se diseñaron tres reglas básicas para el crecimiento del sector privado:

-Regla darwiniana. Para producir había que ser eficiente, y sólo las empresas eficientes quedarían en el mercado. Las empresas ineficientes iban a desaparecer, más aún, era bueno para la economía que quebraran las empresas ineficientes.

-Tanto las ganancias como las pérdidas de las empresas privadas serían privadas. Estoes, bajo ninguna circunstancia el Estado iría al rescate de las empresas privadas en dificultades.

-El sector privado podía endeudarse cuanto quisiera, ya sea en el interior o en el exterior. este era un problema de exclusiva responsabilidad del agente económico que solicitaba los créditos.

También con este modelo se hacen cambios en el sistema de mercados libres:
- El sistema de mercados con precios libres es el mecanismo que asigna los recursos productivos de la manera más eficiente. Esto es efectivo, pero una asignación eficiente de los recursos no tiene nada que ver con una distribución más equitativa de los bienes o una distribución más equitativa de las oportunidades.

El objetivode la eficiencia ha ocupado permanentemente el lugar primordial en el modelo económico aún cuando ello haya implicado un deterioro de la distribución del ingreso.

- No existe el control de precios. Los precios tienen que determinarse por la libre interacción entre la oferta y la demanda, y esto es válido para todos los mercados de la economía.

-El principio de la libertad económica. La libertad económica implícita o explícita en el sistema de mercado libres plantea que los agentes económicos escogen libremente entre una amplia y variada canasta de bienes, lo cual determina lo que el sistema económico va a producir.

- Desde el punto de vista teórico, para que un sistema de mercados funcione de manera (competitivamente) eficiente es requisito importante la atomicidad; esto quiere decir, que haya muchos compradores y vendedores en un mismo mercado.

El sistema de Libre comercio que fue aplicado del modelo económico fueron:

- Una economía pequeña tiene que estar abierta al comercio. El mercado interno es muy chico y el mercado externo permite un mejor aprovechamiento de las economías de escala y el pleno uso de los recursos productivos. 
- Principio de las ventajas comparativas. Una economía abierta al exterior se va a especializar en la producción de bienes intensivos en el recurso del cual posea una mayor abundancia relativa.

- No existe restricción alguna para las importaciones.

- El sistema de aranceles tiene que tener tarifas bajas parejas; de esta manera, se proporciona una protección uniforme a todas las actividades económicas.

- La existencia de un déficit en la Cuenta Corriente de la Balanza de Pagos no constituye un problema. Utilizando el enfoque monetario de la Balanza de Pagos, los problemas de desequilibrios del sector externo están relacionados exclusivamente a la existencia de déficit (o superávit) de la Balanza de Pagos.

El modelo económico también planteaba una apertura del sistema financiero:

- El ideal de este modelo es que haya una perfecta movilidad de capitales financieros. Esto implica que no debiera haber controles cambiarios.

- El modelo económico planteaba que si permite sólo al sector privado que sea quien se endeude en el exterior y se evita que el sector público lo haga, entonces no habría problemas de desequilibrio en el sector externo.

- Mientras sea el sector privado el que solicita los créditos en el extranjero, entonces no debiera haber restricciones de ningún tipo, ya sea con respecto al monto de los créditos o al uso o destino de éstos.

Un principio fundamental para el modelo económico es que en la economía haya reglas neutras; es decir, las reglas tienen que ser iguales para todos.

El enfoque monetario de la balanza de pagos permite entender por qué se optó por una política monetaria restrictiva y no por una devaluación real: esta pudo reforzar el efecto contractivo de la demanda agregada, pero a costa de una mayor inflación. Según el enfoque monetario, el problema de las RIN se resuelve sin necesidad de devaluar pues la medida central es la restricción del crédito interno. La política aplicada mostró una reducida confianza en la agilidad de las exportaciones en el corto plazo frenta a una devaluación real.

El problema central estaba en el déficit fiscal y en la presión que éste ejercía sobre la emisión. Lo que explicaría las medidas iniciales que estuvieron centradas en el terreno fiscal y monetario. Las principales medidas tributarias fueron: el establecimiento de una tasa sobre el precio de los combustibles derivados del petróleo; el aumento del Impuesto General a las Ventas, se eliminaron todas las exoneraciones al pago del mismo (IGV). También en el plano fiscal se eliminaron todas las exoneraciones arancelarias, salvo las de los convenios internacionales, y se estableció un arancel mínimo. Asimismo, se redujo y racionalizó los porcentajes del CERTEX, se eliminó el FENT:

También se hizo incidencia en la dureza de la disciplina fiscal que asumiría el gobierno, afirmando que se iba a gastar, en ningún caso, más de lo que se recibía. Fue por tanto un paquete con efectos fundamentalmente fiscales.

Un aspecto central del programa fue precisamente la decisión de no devaluar y de mantener el nivel del tipo de cambio mediante la compra de divisas de parte del 
Banco Central, ello obedecía a la necesidad de reconstruir el nivel del RIN.

En cuanto a la política cambiaria, se decidió que el Banco Central eliminara el dólar del MUC (Mercado Único de Cambios), y se dejó que el tipo de cambio fuera determinado por el libre.juego de la oferta y la demanda. Para superar la distorsión de precios relativos, se decretó una serie de aumentos de los precios controlados por el gobierno y en las tarifas de servicios públicos. El levantamiento de subsidios y controles condujo también a un enorme aumento de los precios de los alimentos, que anteriormente eran importados a un tipo de cambio subsidiado.

Parte del enfoque empleado radica en que considera la existencia de dólares fuera del circuito oficial. La prioridad durante los primeros días del programa habría sido atraer dichos dólares y recuperar cierta capacidad del manejo monetario más que generarlos mediante un impulso a la actividad exportadora.

Un objetivo central del gobierno era afirmar su autoridad frente a todos los sectores. Por un lado, la elevación de precios públicos permitía al Estado suficiente autonomía respecto del $\mathrm{BCR}$, y así, la posibilidad de cumplir la regla que era condición para obtener recursos externos: no emitir inorgánicamente. Conseguir esos recursos resultaba fundamental para afirmarse ante el país. La decisión de superar las metas de pago de la deuda externa para reisentarse financieramente en el sistema internacional era mucho más difícil si se debía pagar más por dólar comprado, cosa que sucedería en caso de devaluar y que hubiera obligado al Banco Central a emitir más.

Otro rasgo central fue la política salarial. Los reajustes planteados incluían la elevación del salario mínimo de acuerdo a la inflación que se esperaba lograr después del ajuste. En el caso de los trabajadores del sector público, el aumento sería de acuerdo al mismo criterio pero tomando en cuenta la restricción fiscal. En el caso de los salarios privados, su determinación se dejaría al acuerdo entre empresas y trabajadores, eliminándose la indexación automática donde existiera.

La reinserción financiera del Perú en la comunidad financiera internacional y el respaldo del FMI fueron desde el principio elementos indispensables del programa económico del actual gobierno. Con la regularización del pago de las obligaciones corrientes con el Banco Mundial y la continuación del pago de la deuda con el FMI, se iniciarían numerosas conversaciones con ellos, logrando que el BM y el FMI anunciaran su respaldo a dicho programa. El programa económico que el FMI aprobara en agosto de 1991, precisaba los objetivos del mismo para 1991-1992, comprometiéndonos además a consultar con el Fondo cualquier modificación.

En síntesis, el FMI para asegurarse de que cualquier país latinoamericano vaya a cumplir con el programa del FMI y en consecuencia, podrá supuestamente resolver su problema de desequilibrio externo, controla los siguientes cuatro indicadores: el nivel de reservas internacionales, la inflación interna, el volumen de expansión de crédito interno y el déficit fiscal.

\section{2. 'ANÁLISIS DEL MODELO DE DOS BRECHAS}

\subsection{Introducción}

La existencia de una restricción externa o de una seria escasez de divisas, y su impacto sobre la tasa de crecimiento económico, constituyen el foco de atención del modelo de dos brechas. 
El modelo de dos brechas plantea que hay dos elementos que son determinantes en el ritmo de crecimiento económico de una economía latinoamericana (o de un país desarrollado en general):

a).- La maquinaria utilizada para la inversión es casi totalmente importada; además, también se requieren de insumos importados para el proceso productivo, los cuales son muy difíciles de sustituir en el país. La existencia de un estrangulamiento de divisas, en consecuencia, frena el crecimiento, ésta es la brecha externa.

b).- El nivel de inversión está determinado y restringuido por el nivel de ahorro. Luego, la existencia de un déficit de ahorro interno limita la expansión de la inversión y constriñe el crecimiento; ésta es la brecha interna.

Fundamentalmente, el modelo plantea que el flujo de crédito externo ayuda a reducir automáticamente ambas brechas; la mayor disponibilidad de divisas permite financiar las importaciones de maquinaria, $y$ al mismo tiempo, el incremento de ahorro externo complementa el nivel de ahorro interno, y posibilita así la expansión del nivel de inversión.

El principal objetivode la Política Fiscal es el de constituir el ahorro público necesario para financiar la inversión privada exigida por el desarrollo económico, influir en la tasa y estructura de la inversión y el consumo privado en forma congruente con las metas globales y sectoriales de desarrollo y, asegurar al estado los recursos necesarios para la expansión de sus gastos corrientes.

\subsection{Desarrollo del Modelo}

Supongamos que una economía latinoamericana importa toda la maquinaria que utiliza en su proceso productivo, supongamos además, que esta economía está en pleno uso de la capacidad instalada, pero hay desempleo de la fuerza de trabajo.

Sean $*$ Y el incremento del nivel de producción, ${ }^{*} \mathrm{Kel}$ incremento de maquinaria, y k el coeficiente técnico de incremento marginal capital-producto. Luego, cualquier expansión de la producción requerirá un incremento adicional de capital que será:

$$
* \mathrm{~K}=\mathrm{k} * \mathrm{Y}
$$

Sea A el nivel de ahorro total requerido para financiar la expansión de la producción.

$$
\begin{aligned}
& A=k^{*} Y \\
& A=A i+A x
\end{aligned}
$$

Donde:

\section{$\mathrm{Ai}$ : Ahorro interno}

Ax : Ahorro externo

Para simplificar el análisis se supondrá que el ahorro interno Ai es una proporción fija (ã) del ingreso total de la economía Yn; sea $m$ el contenido de insumos importados del nivel de producto Y. Luego.

$$
\begin{aligned}
\mathrm{Ai} & =\tilde{\mathbf{a} Y n} \\
\mathrm{Yn} & =(1-\mathrm{m}) \mathrm{Y} \\
\mathrm{Ai} & =\tilde{\mathbf{a}}(1-\mathrm{m}) \mathrm{Y}
\end{aligned}
$$

Por otra parte, se supondrá que el ahorro externo Ax es una proporción constante $\lambda$ del nivel de producto $Y$; es decir, el flujo de crédito externo es una fracción constante del PBI.

\section{Luego:}

$$
\mathrm{Ax}=\lambda \mathrm{Y}
$$

La expansión del producto, *Y, está limitada por el monto de ahorro total. Luego: 


$$
\begin{gathered}
\mathrm{Y}<\mathrm{A}=\mathrm{Ai}+\mathrm{Ax} \\
\mathrm{K} \quad \mathrm{K} \\
\begin{array}{c}
1[\mathrm{a} \\
\mathrm{K}
\end{array}
\end{gathered}
$$

Sea g la tasa de crecimiento económico; es decir, $\mathrm{g}=* \mathrm{Y} / \mathrm{Y}$, entonces la tasa de crecimiento económico estará restringida por la brecha interna de ahorro:

$$
g<1[a(1-m)+\lambda] \quad \text { ec. } 1
$$

En esta expresión (ec.l) es posible observar que si aumenta el coeficiente de ahorro interno a o si aumenta el coeficiente de ahorro externo $\lambda$, entonces es posible incrementar la tasa de crecimiento $\mathrm{g}$.

La restricción externa proviene de la ecuación de la balanza de pagos; sean Mk y Mi las importaciones de maquinaria y de los insumos productivos. Luego, la expresión del ahorro externo será:

$$
\mathrm{Ax}=\mathrm{Mk}+\mathrm{Mi}-\mathrm{X}
$$

Donde:

$$
\begin{aligned}
& \text { Ax : Ahorro externo } \\
& \mathrm{X}: \text { Exportaciones } \\
& \mathrm{Mk}: \text { Importaciones de maquinarias } \\
& \mathrm{Mi}: \text { Importaciones de insumos }
\end{aligned}
$$

Si se supone que las exportaciones son una proporción constante del nivel de PBI $(\mathrm{x})$, en que $\mathrm{x}=\mathrm{X} / \mathrm{Y}$, se llega que:

$$
\begin{aligned}
& \mathrm{Ax}+\mathrm{X}=\mathrm{Mk}+\mathrm{Mi}-* \mathrm{~K}+\mathrm{mY} \\
& \lambda \mathrm{Y}+\mathrm{xY}=\mathrm{k} * \mathrm{Y}+\mathrm{mY}
\end{aligned}
$$

Considerando que la tasa de crecimiento es $\mathrm{g}=* \mathrm{Y} / \mathrm{Y}$, se tiene que $\mathrm{g}$ estará restringida por la brecha externa de divisas:

$$
\mathrm{g}<1[(\mathrm{x}-\mathrm{m})+\lambda] \quad \text { ec. } 2
$$

En esta expresión (ec. 2) es posible observar que si aumenta el diferencial entre los coeficientes de exportaciones e importaciones; es decir, mientras más grande sea el superávit de la balanza comercial, mayor será la posibilidad de crecimiento económico, algo análogo sucede para mayores coeficientes de crédito externo. Por una parte, la expresión (ec. 2) permite visualizar como coeficientes negativos $\lambda$; es decir, ahorro externo negativo, o transferencia de recursos al exterior, automáticamente disminuyen el nivel de crecimiento g.

Cuando la brecha externa es la dominante, un mayor flujo de divisas para el país es muy importante, por cuanto alivia a restricción externa; en este caso, la rentabilidad de una unidad extra es relativamente muy grande, por cuanto permite expandir la importación de maquinaria, que es el factor limitante del crecimiento, y permite al mismo tiempo financiar la expansión de la inversión.

Nota: Se pueden llegar a los mismos resultados utilizando una expresión más simplificada del Modelo.

$$
\begin{aligned}
& \mathrm{Y}=\mathrm{C}+\mathrm{I}+\mathrm{X}-\mathrm{M} \\
& \mathrm{Y}=\mathrm{C}+\mathrm{A}
\end{aligned}
$$

Restando (1) - (2) Tenemos:

$$
A=1+X-M
$$

$$
(\mathrm{M}-\mathrm{X})=(\mathrm{I}-\mathrm{A})
$$

Brecha Externa Brecha Interna

$$
\begin{aligned}
A & =A i+A x \\
A x & =M-X
\end{aligned}
$$

Reemplazamos en (3) tenemos:

$$
A x=I-A i
$$

Donde: 
Y : Demanda Agregada

$\mathrm{C}$ : Consumo

I : Inversión

$\mathrm{X}$ : Exportaciones

$\mathrm{M}$ : Importaciones

A : Ahorro

$\mathrm{Ai}$ : Ahorro interno

Aip : Ahorro interno público

Aipr: Ahorro interno privado Ax : Ahorro externo

\section{CONCLUSIONES}

1. El crecimiento económico depende directamente del ahorro interno y del superávit comercial. Se puede observar a lo largo de los períodos analizados que las tendencias del crecimiento económico peruano han ido variando a lo largo del tiempo en función de la evolución de las dos variables mencionadas.

2. El modelo de dos brechas sirve para explicar el crecimiento, en función de restricciones internas y externas, por lo que es un modelo apropiado y aplicable a economías subdesarrolladas cuya desarticulación se expresa en la existencia de ambas brechas.

3. En la década de los ochenta el crecimiento económico peruano estuvo determinado por la vigencia de las dos brechas (brecha interna y brecha externa), que actuaban simultáneamente y de manera simétrica; es decir, que ambas brechas se agravaron y mostraban tendencias dinámicas similares. Este modelo ayuda a explicar, entonces, la crisis por la que atravesaba la economía peruana.

4. En la década de los noventa se aplican políticas económicas distintas, orientadas fundamentalmente al control y superación de la crisis heredada: el proceso de hiperinflación se combinaba con el estancamiento económico. Como resultado de las medidas estructurales y de políticas aplicadas la economía peruana presenta una tendencia de crecimiento diferente; tendencias que hace prever una superación de la crisis pasada, sin embargo, las brechas manifiestan tendencias asimétricas, es decir, la brecha interna es resuelta mientras que la brecha externa tiende a incrementarse peligrosamente. 
5. En lo que resta a la presente década, el crecimiento de la economía peruana que presenta tendencias de recuperación podría tener una limitante o una seria restricción determinada por la importancia de la brecha externa.

\section{BIBLIOGRAFIA}

BANCOCENTRALDERESERVA DEL PERÚ Memoria Anual. Años desde 1985 hasta 1997, Lima.

BOLOÑA, C.

1993. "Cambio de Rumbo"

Lima.

CUBA,E. 1995. ¿Está recalentada la economía? Un cálculo. En Revista: Actualidad Económica Nro. 166, Lima, agosto.

FRANCKE, $P$.

1996. Crecimiento, sectores económicos y pobreza. En Revista: Moneda. Año 8.

Nro. 94-95. Lima, abril - mayo.

FOXLEY,A.

1982. "Experimentos neoliberales en América Latina". Colección Estudios CIEPLAN

7. Nro. Especial, Santiago.

IGUIÑEZ, J. - BASAY, R. - RUBIO, M.

1993. "Los ajustes Perú 1975-1992".

Fundación Friedrich Ebert, Lima.

INSTITUTO NACIONAL DE ESTADÍSTICA Compendios Estadísticos. Años desde 1980 hasta 1997, Lima.

\section{LEÓN MENDOZA, J.}

1995. "Restricción externa al crecimiento: ¿Hasta cuándo?. En Revista: Actualidad Económica. Nro. 169, Lima. noviembrediciembre.

MEDINA, A.

1995."Pobreza,crecimiento y desigualdad:
Perú 1994". En Revista: Moneda Año7, Lima, enero- febrero.

MELLER,P.

1987. "Enfoques de la cuenta corriente". En Revista de la Cepal. Nro. 32, Santiago, agosto.

MENDOZA BELLIDO, $w$.

1995. "La economía peruana: ¿Fría o

Caliente?" En Revista: Actualidad Económica Nro. 164, Lima. mayo-junio.

MOREYRA, M.

1990. "Hacia la estabilización y el crecimiento". Universidad del Pacífico, Lima.

PAREDES LANATHA, C. - SACHS. J.

1990. "Estabilización y crecimiento en el Perú: Una propuesta independiente".GRADE, Lima, 1990.

SAMUELSON P/NORDHAUS, $\mathrm{W}$.

1988. "Economía". Editorial Mc Graw Hill, México.

SCHULDT, J.

1988. "Políticas heterodoxas de estabilización en América Latina". Apuntes 22, Santiago, Primer Semestre.

TELLO, Mario

1992. "Mecanismos hacia el crecimiento económico", Lima.

TOLEDO, Alejandro 1989. "¿Cómo financiar el crecimiento?". ESAN, Lima.

TOMATIS CHIAPPE, $\mathrm{B}$.

1993. Crecimiento económico en el Perú. Causas y perspectivas. En Revista: Moneda. Año 5. Nro. 63-64, Lima, octubre.

VEGA-CENTENO, M. 1989. Crecimiento económico. En Revista: Economía. Vol. 24. Nro. 18, Lima, diciembre. 